\title{
An application of a semi-hidden Markov model in wireless communication systems
}

\author{
Shaghayegh Kordnoori ${ }^{1}$ [D $\cdot$ Hamidreza Mostafaei $^{2} \cdot$ Mohammadhassan Behzadi $^{1}$
}

Received: 1 February 2019 / Accepted: 5 March 2019 / Published online: 16 March 2019

(c) The Author(s) 2019

\begin{abstract}
Stochastic processes are approved presentation of real systems which its development in space or time can be supposed as random. A semi-hidden Markov model as a type of stochastic processes is a modification of hidden Markov models with states that are no longer totally unobservable and are less hidden. This mathematical model is employed for modeling data sequences with long runs, memory and statistical inertia. In this article, we investigate the theory of the semi-hidden Markov model along with its parameter estimation and order estimation methods. Moreover, the proposed model is applied to model the error traces generated by the wireless channels. A new Markov-based trace analysis algorithm is suggested to divide a non-stationary network error trace into stationary parts. By means of the best semi-hidden Markov model and fitting probability distribution, we would be able to model these parts accurately. Calculating the information measure criteria and the autocorrelation function by running the modified Baum-Welch algorithm several times help us to find the optimal order of the semi-hidden Markov model.
\end{abstract}

Keywords Stochastic processes $\cdot$ Semi-hidden Markov model $\cdot$ Order estimation $\cdot$ Wireless communication

Mathematics Subject Classification $60 \mathrm{~J} 20 \cdot 60 \mathrm{~K} 15 \cdot 90 \mathrm{~B} 18$

\section{Introduction}

A stochastic process is a probability model employed for describing the evolution in time of a random event. The hidden Markov models (HMMs) make a profitable and flexible class of stochastic processes which have been used satisfactorily in a broad range of applied problems. The hidden Markov model (HMM) is an extension of a Markov chain whose states are hidden. It is a doubly stochastic model $\left\{\left(H_{k}, O_{k}\right)\right\}$, where $\left\{H_{k}\right\}$ denotes the hidden state sequence which is a finite-state Markov chain. Given $\left\{H_{k}\right\}$, the $O_{k}$ is conditionally independent and the conditional distribution of it relies on $\left\{H_{k}\right\}$ only through $H_{n}$. The (HMMs) have many

Shaghayegh Kordnoori

shaghayegh.kordnoori@srbiau.ac.ir

$\triangle$ Hamidreza Mostafaei

h_mostafaei@iau-tnb.ac.ir

1 Department of Statistics, Science and Research Branch, Islamic Azad University, Tehran, Iran

2 Department of Statistics, North Tehran Branch, Islamic Azad University, Tehran, Iran applications in different fields such as speech recognition [1], hand gesture recognition [2], source coding [3], seismic hazard assessment [4], traffic prediction [5],wireless network [6-8], protein structure prediction [9] and finance [10]. The semi-hidden Markov models (SHMMs) are stochastic models related to HMMs. They are discussed in [11, 12], recently. A principal characteristic of these models is the involvement of statistical inertia which admits the generation, and analysis of observation symbol contains frequent runs. The SHMMs cause a substantial reduction in the model parameter set. Therefore in most cases, these models are computationally more efficient models compared to HMMs in most cases. As long stretches of error-free transmission exist in wireless channels, the corresponding runlength vector is greatly shorter in length than the original binary data. Hence, the simulation runtime decreases considerably.

In this paper, the definition and the modified Baum-Welch algorithm for the parameter estimation of the SHMM are given first and next, the order estimation benchmarks of these models are discussed. Next, we present an application of the SHMM in wireless communication for modeling 
the error sequence generated in the CDMA system. Finally, conclusions are given.

\section{The semi-hidden Markov model}

The SHMMs are the advanced types of stochastic models connected to HMMs. They can model the behavior of symbolic sequences with inertia, memory and long runs. If we know the state of changing points, given these sequences it is probable to realize the sequence of states. Hence, the semi-hidden Markov model (SHMM) is not absolutely hidden. The SHMMs work by altering among distinct states and generating symbols of the alphabet in the same way as the HMMs. The input sequence is in terms of a runlength vector, and therefore, the length of the input sequence is extremely reduced, especially when the sequences involve long stretches of identical symbols.

In this section, we represent a modified version of the forward-backward (BWA) that is employed to estimate the parameters of the SHMMs. Figure 1 indicates the flowgraph of the SHMM.

The parameters of SHMMs can be estimated by the algorithms similar to the Baum-Welch Algorithm (BWA). Suppose $\mathbf{x}_{1}^{T}$ be the observation sequence which is explained by the sequence of different observation values $X_{1}, X_{2}, \ldots, X_{\mathrm{N}}$ and the number of repetitions $m_{1}, m_{2}, \ldots, m_{\mathrm{N}}$. Let $f\left(\mathbf{x}_{1}^{T}\right)$ be a process multidimensional distribution. We can write the likelihood function as

$g\left(\mathbf{x}_{1}^{T}, \tau\right)=h\left(\boldsymbol{v}_{1}^{N}, \tau\right)=\pi \prod_{i=1}^{N} \mathbf{P}^{m_{i}}\left(X_{i}, \tau\right) \mathbf{1}$

where $v_{i}=\left(X_{i}, m_{i}\right), N$ is the number of subsegments containing identical observations and $\tau$ is the model parameter vector which can be omitted in the equations in which its actual value is not important. To calculate $\mathbf{P}^{m}(X)$ using the fast exponentiation algorithm, we express the power as a binary number

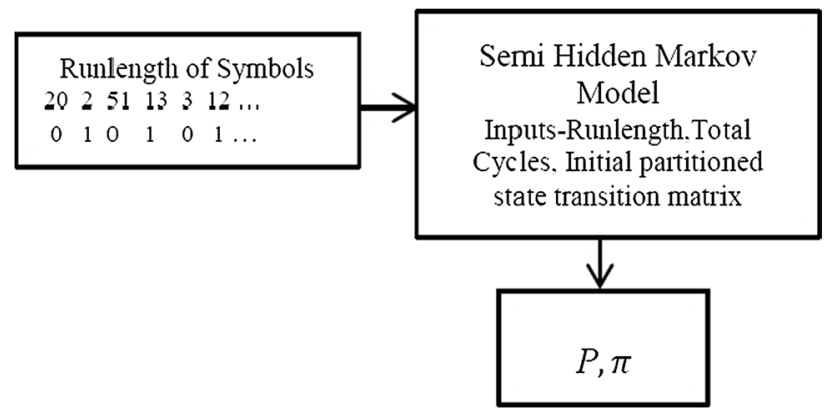

Fig. 1 The semi-hidden Markov flowgraph $m=b_{1}+2 b_{2}+\cdots+2^{k-1} b_{k}$

$\mathbf{P}^{m}(X)$ is found as a result of the following recursion:

Algorithm:

Initialize:

$\mathbf{R}_{\mathbf{1}}=\mathbf{P}(X) \mathbf{Q}_{0}=\mathbf{I}$

For $i=1,2, \ldots, k$

Begin

$\mathbf{Q}_{i}=\mathbf{Q}_{i-1} \mathbf{R}_{i}^{b_{i}}, \quad \mathbf{R}_{i+1}=\mathbf{R}_{\mathbf{i}}^{2}$

End

We obtain the fast forward algorithm

$\boldsymbol{\alpha}_{i}=\boldsymbol{\alpha}_{i-1} \mathbf{R}_{i}^{b_{i}}, \quad \mathbf{R}_{i+1}=\mathbf{R}_{\mathbf{i}}{ }^{2}, i=1, \ldots, k$

which can be used to calculate the forward probabilities

$\boldsymbol{\alpha}\left(\boldsymbol{v}_{1}^{n_{t}}, \tau\right)=\boldsymbol{\pi} \prod_{i=1}^{n_{t-1}} \mathbf{P}^{m_{i}}\left(X_{i}, \tau\right)$

where $n_{t}$ is the number of different observation series up to time $\mathrm{t}$.

Similarly, we obtain the fast backward algorithm

$\boldsymbol{\beta}_{i}=\mathbf{R}_{i}^{b_{i}} \boldsymbol{\beta}_{i-1}, \quad \mathbf{R}_{i+1}=\mathbf{R}_{\mathbf{i}}^{2}, i=1, \ldots, k$

for calculating the backward probabilities

$\boldsymbol{\beta}\left(\boldsymbol{v}_{1}^{N}, \tau\right)=\prod_{i=n_{t}}^{N} \boldsymbol{P}^{m_{i}}\left(X_{i}, \tau\right) \mathbf{1}$

We have

$h\left(\boldsymbol{v}_{1}^{N}, \tau\right)=\boldsymbol{\pi}_{X_{1}} \prod_{i=1}^{N-1}\left[\mathbf{P}_{X_{i} X_{i}}^{m_{i}-1} \mathbf{P}_{X_{i} X_{i+1}}\right] \mathbf{P}_{X_{N} X_{N}}^{m_{N}-1} \mathbf{1}$

To indicate the forward and backward algorithm for calculating $h\left(\boldsymbol{v}_{1}^{N}, \tau\right)$ in block-vector form, we define

$\boldsymbol{\alpha}_{0}\left(\boldsymbol{v}_{1}^{t}, \tau\right)=\boldsymbol{\pi}_{X_{1}} \prod_{i=1}^{t-1}\left[\mathbf{P}_{X_{i} X_{i}}^{m_{i}-1} \mathbf{P}_{X_{i} X_{i+1}}\right]$

$\boldsymbol{\alpha}\left(\boldsymbol{v}_{1}^{t}, \tau\right)=\boldsymbol{\pi}_{X_{1}} \prod_{i=1}^{t-1}\left[\mathbf{P}_{X_{i} X_{i}}^{m_{i}-1} \mathbf{P}_{X_{i} X_{i+1}}\right] \mathbf{P}_{X_{t} X_{t}}^{m_{t}-1}$

These vectors can be analyzed recursively by the following forward algorithm:

$$
\begin{aligned}
\boldsymbol{\alpha}_{0}\left(\boldsymbol{v}_{1}^{0}, \tau\right) & =\boldsymbol{\pi}_{X_{1}}, \boldsymbol{\alpha}\left(\boldsymbol{v}_{1}^{t}, \tau\right) \\
& =\boldsymbol{\alpha}_{0}\left(\boldsymbol{v}_{1}^{t}, \tau\right) \mathbf{P}_{X_{t} X_{t}}^{m_{t}-1} \boldsymbol{\alpha}_{0}\left(\boldsymbol{v}_{1}^{t+1}, \tau\right) \\
& =\boldsymbol{\alpha}\left(\boldsymbol{v}_{1}^{t}, \tau\right) \mathbf{P}_{X_{t} X_{t+1}}
\end{aligned}
$$


The backward variable is defined as

$\boldsymbol{\beta}_{0}\left(\boldsymbol{v}_{t}^{N}, \tau\right)=\prod_{i=t}^{T}\left[\mathbf{P}_{X_{i-1} X_{i}} \mathbf{P}_{X_{i} X_{i}}^{m_{i}-1}\right] \mathbf{1}$

$\boldsymbol{\beta}\left(\boldsymbol{v}_{t}^{N}, \tau\right)=\mathbf{P}_{X_{t} X_{t}}^{m_{t}-1} \prod_{i=t+1}^{T}\left[\mathbf{P}_{X_{i-1} X_{i}} \mathbf{P}_{X_{i} X_{i}}^{m_{i}-1}\right] \mathbf{1}$

We obtain the block-form backward algorithm as

$$
\begin{aligned}
\boldsymbol{\beta}_{0}\left(\boldsymbol{v}_{N+1}^{N}, \tau\right) & =\mathbf{1}, \boldsymbol{\beta}\left(\boldsymbol{v}_{t}^{N}, \tau\right) \\
& =\mathbf{P}_{X_{t} X_{t}}^{m_{t}-1} \boldsymbol{\beta}_{0}\left(\boldsymbol{v}_{N}^{t+1}, \tau\right) \boldsymbol{\beta}_{0}\left(\boldsymbol{v}_{t}^{N}, \tau\right) \\
& =\mathbf{P}_{X_{t} X_{t+1}} \boldsymbol{\beta}\left(\boldsymbol{v}_{t+1}^{N}, \tau\right)
\end{aligned}
$$

Next, suppose that $q_{t}$ is the state at time $t$. In order to estimate the model $\Lambda$, define

$\Gamma_{t}(i, j)=\operatorname{Pr}\left(q_{t}=i, q_{t+1}=j \mid \mathbf{x}_{1}^{T}, \Lambda\right)$

This can be described in relation to the forward and backward variables as

$\Gamma_{t}(i, j)=\frac{\alpha_{t}(i) \Lambda_{X_{N} X_{N+1}} \Lambda_{X_{N} X_{N+1}}^{m\left(X_{N+1}\right)-1} \beta_{t}(j)}{P\left(\mathbf{x}_{1}^{T} \mid \Lambda\right)}$

$\Gamma_{t}(i, j)=[0]$ unless $i=X_{N}$ and $j=X_{N+1}$ where [0] represents a matrix consisting of all zeros. The probability transition matrix $\boldsymbol{P}$ is estimated by calculating the expected number of transition from $i$ to $j$ as $\sum_{t=1}^{N-1} \Gamma_{t}(i, j)$.

One of the most important problems with SHMMs is order estimation. The information criteria are the rectification of the parameters and uncertainty in the model. They are based on the maximum log-likelihood estimate $(L)$ of the model. These criteria try to choose an order of the model with small generalization error. Three common criteria for the mentioned purpose are as follows in which $k$ and $n$ are the numbers of parameters and observations, respectively:

1. The Akaike information criterion (AIC) was proposed by Akaike (1973) [13]. It is defined as

$$
\mathrm{AIC}=-2 \log (L)+2 k
$$

2. The Bayesian information criterion (BIC) was introduced by Schwarz (1978) [14]. It is computed as

$$
\mathrm{BIC}=-2 \log (L)+k \log n
$$

3. The Hannan-Quinn information criterion (HQC) is calculated as

$$
\mathrm{HQC}=-2 \log (L)+2 k \log (\log (n))
$$

\section{An application of the SHMM in wireless communication}

Modeling wireless communication errors is substantial for simulation-based performance assessment of network protocols or for utilizing information about these error characteristics within a protocol. Discrete channel models (DCMs) were employed in wireless systems such as code-division multiple access (CDMA) [15], orthogonal frequency division modulation (OFDM) [16] and global system for mobile communication (GSM) [17]. HMMs are dominant tools with high accuracy which employed as the discrete channel model (DCM) for modeling stochastic processes. These models are applied for precise simulation of errors in wireless systems [18-22]. In this section, the SHMM is used for modeling the errors of the CDMA as the DCM. The order estimation is profitable for interpreting the model. Moreover, it is vital to ensure stability. The estimation of the order of the HMM was investigated in [23]. They discussed the optimal order estimation of the SHMM for the error sequences generated by the CDMA.

\section{The CDMA specification}

The CDMA is a channel access method employed by various radio communication technologies. It has considerable advantages over other similar technologies. CDMA proposes a high put-through rate and can prevail over resilient interference which leads to an increase in the system capacity. The importance of the CDMA makes its modeling necessary to assess and analyze the error control pattern. The superiority of the CDMA makes the modeling necessary for assessing and analyzing the error control pattern. These errors are yielded by comparing the detected with desired symbols and can be shown by the SHMM. Refer to [24, 25] for more details about CDMA. The block diagram for the SHMM-based simulation model of a CDMA link is given in Fig. 2. In Fig. 2, a multiuser CDMA system is shown which is considered to increase the capacity of the system. In this system, $K$ users have considered which one user is the desired one and the other $K-1$ users are the interfering users. Both have the same blocks. All these users then transmit simultaneously in the same frequency band and are distinguished at the receiver by the user specific spreading code. The interfering users appear as interference to the desired user because of nonzero cross-correlation values between the spreading codes. All the desired user, interfering users and additive noise combine in the wireless channel.

\section{Analysis of the CDMA system with the SHMM}

The simulation of a CDMA system is performed in this section. It includes interference and thermal noise. It operates in a multipath/fading environment. The Rayleigh fading 


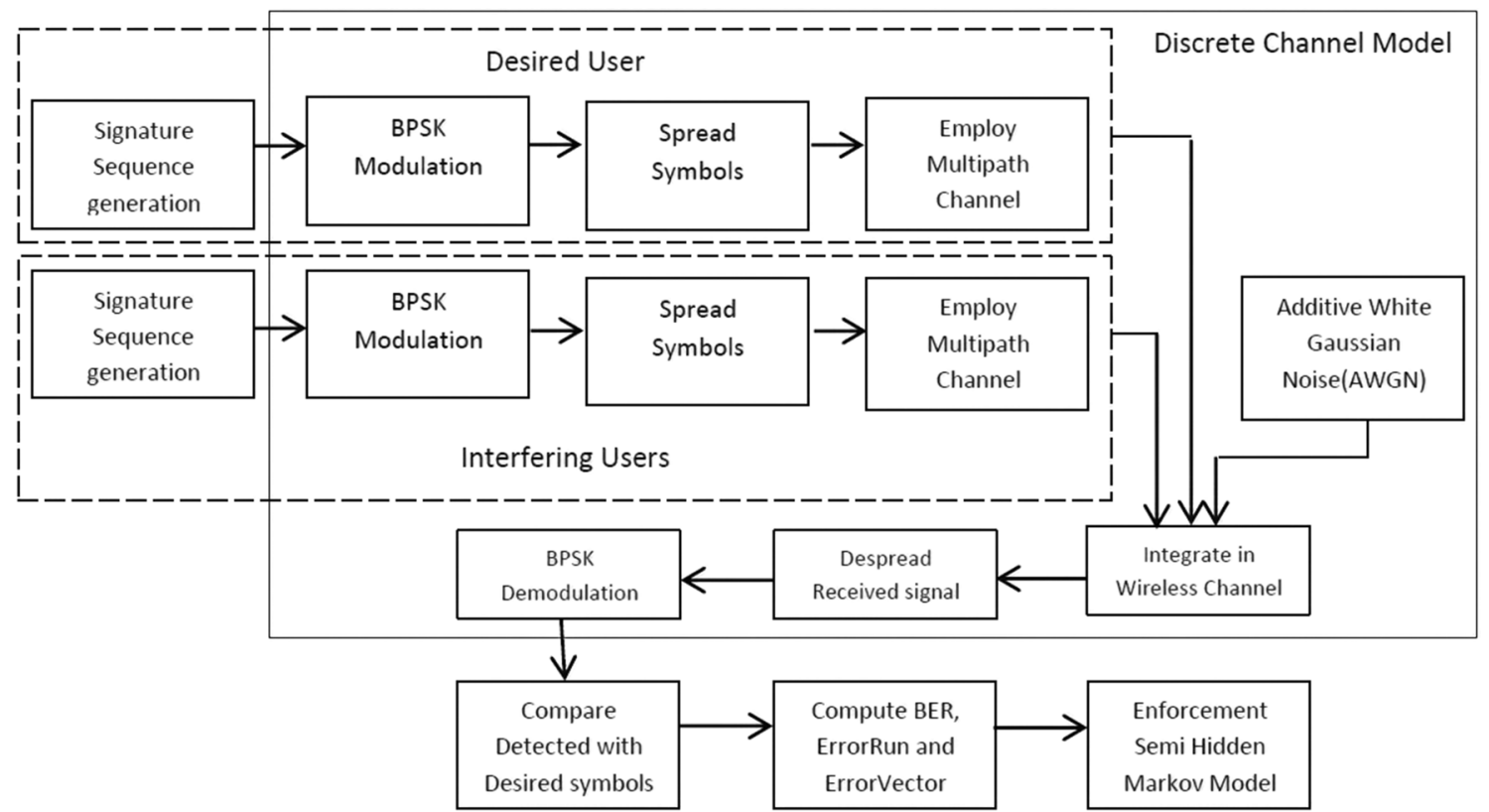

Fig. 2 Block diagram of the SHMM implementation for the CDMA

on each multipath component is exhibited. Each MAI signal uses the same PN-sequence as the signature/spreading sequence. BPSK modulation is regarded, and pulse shaping is ignored. All MAI signals and the desired signal are chip synchronized at the receiver. The input parameters are supposed to be $\mathrm{K}$ factordB $=0, \mathrm{SF}=63$, Number of Interferers $(\mathrm{NoI})=30$, and Mpathdelay $=\left[\begin{array}{ll}2 & 25 \\ 85\end{array}\right]$ with 12,000 symbols are supposed. The run test is performed, and the $p$ value is obtained 0.295. It indicates that at a significance level of $\alpha=0.05$, the error trace produced from the waveform level simulation is randomly distributed. The slow fading causes this non-stationary. Moreover, existence of the inertia which is the characteristic of SHMM is evident. The original error trace is separated into lossy and error-free traces. The first trace is comprised of $1^{\prime} s$ and $0^{\prime} s$ with the first element being a 1 , and the second one contains zeros in runlength vector form. Therefore, two random processes with space $S=\{0,1,2, \ldots\}$ are as follows:

- $\left\{L_{n} \mid n \geq 0\right\}$ : The lossy state length process, where $L_{n}$ shows the length of the $n$th state.

- $\left\{G_{n} \mid n \geq 0\right\}$ : The error-free state length process, where $G_{n}$ shows the number of elements in the $n$th error-free state.

The length of a lossy trace is determined by the change of state constant $(C)$ which is the sum of the mean and standard deviation of the error burst runlengths. The run test is performed on the lossy trace, and the $p$-value (two-tailed) smaller than 0.0001 admits the acceptance of the stationary hypothesis at the significance level of 0.05. Different SHMMs are trained to model the lossy trace. It is observed that the SHMM with 2 states is the best model. The sample autocorrelation function (ACF) comparison of the lossy data trace with 2 to 6 state SHMM is exhibited in Fig. 3; Furthermore, the MSE ACFs are calculated for judging simpler (Table 1).

The criteria AIC, BIC and HQC of the different SHMMs are given in Table 2. It is obvious that all these measures choose 2-state SHMM as the best model. The best parameters of this model are estimated as follows:

$A=\left(\begin{array}{cc}0 & 1 \\ 0.1271 & 0.8729\end{array}\right) \quad B=\left(\begin{array}{ll}0.2692 & 0.7308\end{array}\right)$

The best fitting distributions for the runlength of errorfree and lossy traces are the generalized Pareto distribution (GPD) and two-parameter Gamma distribution, respectively. Figures 4 and 5 indicate the cumulative distribution function (CDF) for the $G_{n}$ and $L_{n}$ along with their empirical cumulative distribution functions. The CDF forms of (GPD) and $\operatorname{gamma}(\alpha, \beta)$ distributions are as follows:

$F(x)= \begin{cases}1-\left(1+k \frac{(x-\mu)}{\sigma}\right)^{-\frac{1}{k}}, & \text { if } k \neq 0 \\ 1-\exp \left(-\frac{(x-\mu)}{\sigma}\right), & \text { if } k=0\end{cases}$

with domain:

$\begin{cases}\mu \leq x \leq \infty, & \text { for } k \geq 0 \\ \mu \leq x \leq \mu-\frac{\sigma}{k}, & \text { for } k<0\end{cases}$

and 
Fig. 3 The ACF comparison of lossy data with different states of SHMM
Table 1 MSE ACFs of different SHMMs for the lossy trace

$F(x)=\frac{\Gamma_{x}(\alpha)}{\Gamma(\alpha)}$

Table 2 AIC, BIC and HQC values of different SHMMs for the lossy trace

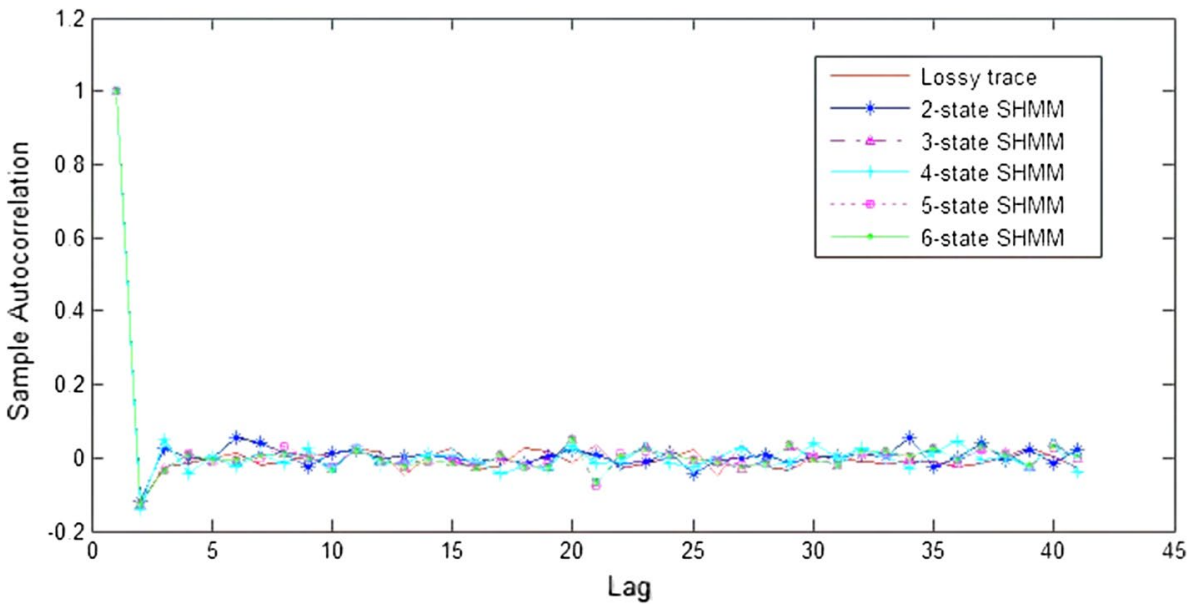

where $\Gamma$ is the Gamma function and $\Gamma_{x}$ is the incomplete Gamma function.

The parameters for these best distributions are $k=0.05811, \sigma=5.3891, \mu=1.6524$ for the (GPD) and $\alpha=2.1861, \beta=0.70809$ for the Gamma distribution.

The binary data are produced by generating lossy trace utilizing the 2-state SHMM and by generating runlengths of the error-free trace from the GPD as follows:

1. Choose the number of lossy and error-free frames $(N)$ to generate in the artificial trace.

\begin{tabular}{llllll}
\hline Model & $\mathrm{k}$ & Log-likelihood & AIC & BIC & HQC \\
\hline 2-state SHMM & 4 & 305.3651 & 618.7302 & 624.0041229 & 614.8977144 \\
3-state SHMM & 9 & 305.3651 & 628.7302 & 640.5965263 & 620.1071073 \\
4-state SHMM & 16 & 305.1949 & 642.3898 & 663.4854912 & 627.0598575 \\
5-state SHMM & 25 & 305.1932 & 660.3864 & 693.3484175 & 636.4333648 \\
6-state SHMM & 36 & 305.1948 & 682.3896 & 729.8549052 & 647.8972293 \\
\hline
\end{tabular}

Fig. 4 The error-free state runlength distribution

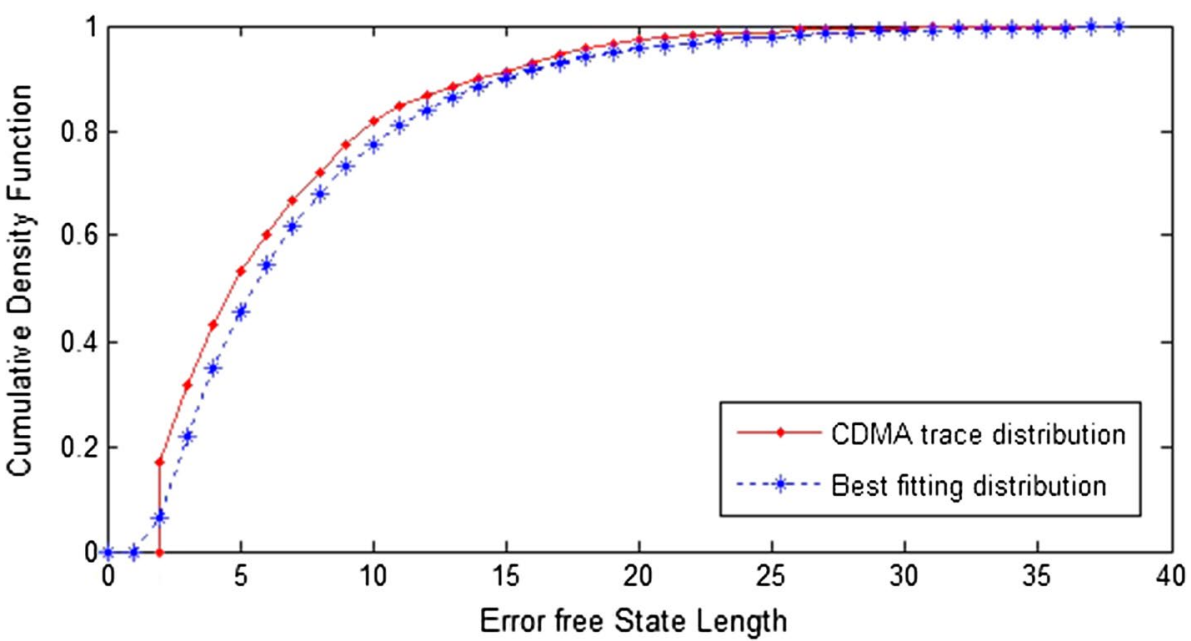


2. Identify the length of an error-free state $\left(g_{\text {len }}\right)$ from the $G_{n}$ using the inverse CDF method.

3. Generate a sequence of zeros $\left(g_{\text {len }}\right)$ to make an error-free burst.

4. Identify the length of lossy state $\left(l_{l e n}\right)$ from the $L_{n}$ using the inverse CDF method.

5. Generate a sequence of $\left(l_{l e n}\right)$ burst which is either lossy or error-free frames based on 2-state SHMM.

6. Compound the two sequences to the artificial trace.
7. Stop if all $N$ frames have been generated, else return to step 2. The original and artificial error traces are compared according to the ACF in Fig. 6. It is evident that the two plots are matched. Moreover, from Fig. 7, it can be concluded that the distributions of error-free intervals for these two sequences are in the same manner which validates the accuracy of our mathematical model.
Fig. 5 The lossy state runlength distribution

Fig. 6 The ACF comparison of the original and artificial error traces

Fig. 7 The error-free interval distribution comparison of the original and artificial error traces
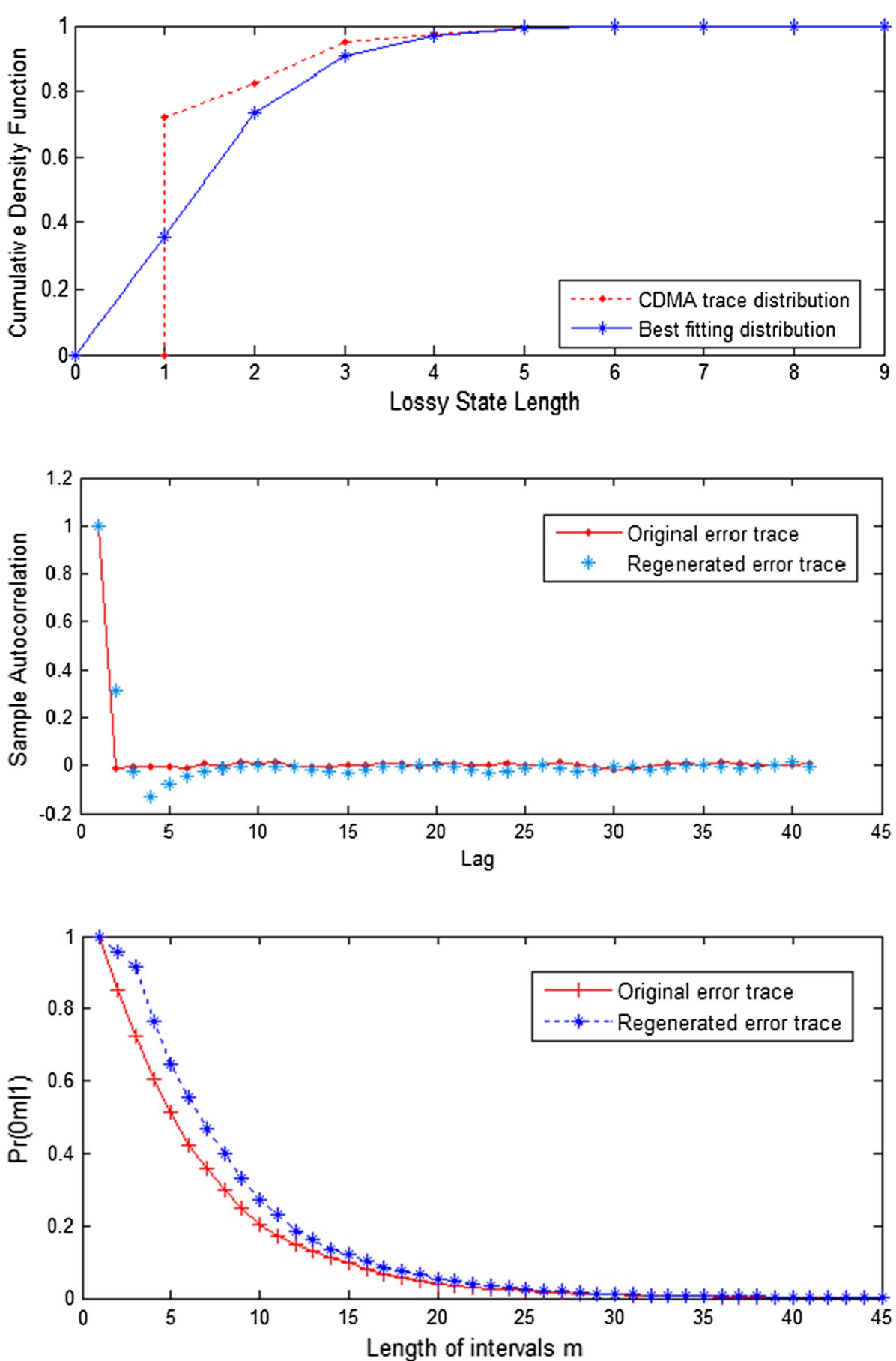


\section{Conclusion}

Analyzing the network protocol and performance depends on the methods of modeling and simulating channel conditions. Wireless channels usually face bursty errors. In this paper, we demonstrated that the SHMM as a discrete channel model can accurately model the errors of a CDMA system. The simulation contained the effects of multipath, additive white Gaussian noise and multiple access interference to generate the error sequence. The original error trace exhibited a non-stationary performance. Therefore, we divided the data into two lossy and error-free traces to obtain stationary behavior. The SHMM was used to model the lossy trace. The AIC, BIC, HQC and sample autocorrelation criteria were employed to find the best model as a 2-state SHMM. The best fitting of a runlength of the lossy and error-free trace was the two-parameter Gamma distribution and the generalized Pareto distribution, respectively. An artificial binary error trace was generated by integrating the 2-state (SHMM) and generalized Pareto distribution according to the algorithm we explained. The original error trace matched closely with the artificial one according to the sample autocorrelation function. All in all, the semi-hidden Markov model is a reliable stochastic model for modeling symbolic sequences with long runs and statistical inertia. It has become a precise mathematical feature to model the error traces generated by wireless channels.

Open Access This article is distributed under the terms of the Creative Commons Attribution 4.0 International License (http://creativeco mmons.org/licenses/by/4.0/), which permits unrestricted use, distribution, and reproduction in any medium, provided you give appropriate credit to the original author(s) and the source, provide a link to the Creative Commons license, and indicate if changes were made.

\section{References}

1. Champion, C., Houghton, S.M.: Application of continuous state hidden Markov models to a classical problem in speech recognition. Comput. Speech Lang. 36, 347-364 (2016)

2. Premaratne, P., Yang, S., Vial, P., Ifthikar, Z.: Centroid tracking based dynamic hand gesture recognition using discrete hidden Markov models. Neurocomputing 228(C), 79-83 (2017)

3. Yang, H., Qing, L., He, X.: Joint source-channel coding for first-order hidden Markov source. In: International Conference on Computational Intelligence and Communication Networks (CICN), (2015)

4. Votsi, I., Limnios, N., Tsaklidis, G., Papadimitriou, E.: Hidden Markov models revealing the stress field underlying the earthquake generation. Phys. A Stat. Mech. Appl. 392(13), 2868-2885 (2013)

5. Qi, Y., Ishak, S.: A hidden Markov model for short term prediction of traffic conditions on freeways. Transp. Res. Part C Emerg. Technol. 43(1), 95-111 (2014)

6. Iiyas, M.U., Radha, H.: Modeling, estimating and predicting the packet-level bit error rate process in IEEE802.15.4 LR-WPANS using hidden Markov models. In: 43rd Annual Conference on Information Sciences and Systems ( 2009)

7. Salih, O.S., Wang, C.-X., Laurenson, D.I.: Three layered hidden Markov models for binary digital wireless channels. In: IEEE International Conference on Communications, 2009, ICC '09. Dresden, Germany ( 2009)

8. Gani, M.D.O., Sarwar, H., Rahman, C.M.: Prediction of state of wireless network using Markov and hidden Markov model. J. Netw. 4(10), 976-984 (2009)

9. Kamal, M.S., Chowdhury, L., Khan, M.I., Ashour, A.S., Tavares, J.M.R.S., Dey, N.: Hidden Markov model and Chapman Kolmogrov for protein structures prediction from images. Comput. Biol. Chem. 68, 231-244 (2017)

10. Sin, T.K., Ching, W.K., Fung, E.S., Ng, M.K.: Extracting information from SPOT interest rates and credit rating using double higher-order Hidden markov models. Comput. Econ. 29(3), 69-102 (2007)

11. Gomez-Lopera, J.F., Martinez-Aroza, J., Roman-Roldan, R., Roman-Galvez, R.: The evaluation problem in discrete semi-hidden Markov models. Math. Comput. Simul. 137, 350-365 (2017)

12. Roman-Galvez, R., Roman-Roldan, R., Martinez-Aroza, J., Gomez-Lopera, J.F.: Semi hidden Markov models for generation and analysis of sequences. Math. Comput. Simul. 118, 320-328 (2015)

13. Akaike, H.: Information theory as an extension of the maximum likelihood principle. In: Second International Symposium on Information theory Akademia Kiado Budapest. Budapest (1973)

14. Schwarz, G.: Estimating the dimension of a model. Ann. Stat. 6(2), 461-464 (1978)

15. Kuczynski, P., Rigolle, A., Gerstacker, W.H., Huber, J.B.: Hidden Markov modeling of error patterns and soft outputs for simulation of wideband CDMA transmission systems. Int. J. Electr. Commun. (AEU) 58(4), 256-267 (2000)

16. Lin, K.-Y., Lin, H.-P., Tseng, M.-C.: Link adaptation of MIMOOFDM systems using hidden Markov model for high speed railway. In: 16th Asia-Pacific Conference on Communications (APCC). Auckland, New Zealand ( 2010)

17. Almudena, K., Zhao, B.Y., Ludwig, A.D., Joseph, R.: A Markovbased channel model algorithm for wireless networks. Wirel. Netw. 9(3), 189-199 (2003)

18. Guzelarsian, B., Dippold, M., Paul, M.: Efficient and automatized error pattern modelling with hidden markov models in digital communication. Int. J. Electr. Commun. 66(5), 417-432 (2012)

19. Balaban, P., Li, D., Turin, W.: Performance evaluation of the forward link of a CDMA cellular system. In: Military Communications Conference Proceedings. IEEE (1999)

20. Turin, W.: Simulation of error source in digital channels. IEEE J. Sel. Areas Commun. 6(1), 85-93 (1988)

21. Srinivas, S., Shanmugan, K.S.: Form-hmm, a forward-only realtime modifed hidden markov modeling algorithm for tracking bursty digital channels. In: Fifth IEEE International Workshop on Computer-Aided Modeling, Analysis, and Design of Communication Links and Networks, CAMAD '94 (1994)

22. Turin, W., Van Nobelen, R.: Hidden Markov modeling of fading channels. IEEE J. Sel. Areas Commun. 16(9), 1809-1817 (1998)

23. Gassiat, E., Boucheron, S.: Optimal error exponents in hidden Markov models order estimation. IEEE Trans. Inf. Theory 49(4), 964-980 (2003)

24. Groe, J.B., Larson, L.E.: CDMA Mobile Radio Design. Artech House, Inc, Norwood (2000)

25. Prasad, R.: CDMA for Wireless Personal Communications. Artech House, Inc, Norwood (1996)

Publisher's Note Springer Nature remains neutral with regard to jurisdictional claims in published maps and institutional affiliations. 